\title{
Severe Hyponatremia Masking Central Diabetes Insipidus in a Patient with a Lung Adenocarcinoma
}

\author{
Eduardo Terán Brage ${ }^{a, b} \quad$ Manuel Heras Benito $^{c}$ \\ Marta Belén Navalón Jiménez ${ }^{a, b}$ Rosario Vidal Tocino ${ }^{a, b}$ \\ Edel del Barco Morillo ${ }^{a}$ b Emilio Fonseca Sánchez ${ }^{a, b}$ \\ aDepartment of Medical Oncology, University Hospital of Salamanca, Salamanca, Spain; \\ bInstitute for Biomedical Research of Salamanca (IBSAL), Salamanca, Spain; 'Department of \\ Nephrology University Hospital of Salamanca, Salamanca, Spain
}

\section{Keywords}

Lung adenocarcinoma - Central diabetes insipidus · Inappropriate antidiuretic hormone secretion syndrome $\cdot$ Immunotherapy

\begin{abstract}
Altered natremia is a common electrolyte disorder in clinical practice and a paraneoplastic manifestation. The syndrome of inappropriate antidiuretic hormone secretion is the first diagnostic suspicion in a patient with cancer and hyponatremia, although entities such as adrenal insufficiency primary or secondary to metastatic involvement must be taken into account. Likewise, immunorelated endrocrinopathies such as hypophysitis have been reported after the introduction of checkpoint inhibitors. A 46-year-old man diagnosed with metastatic adenocarcinoma of the lung with severe hyponatremia $(111 \mathrm{mmol} / \mathrm{L})$ consulted due to altered level of consciousness. The initial cranial CT scan did not reveal pituitary brain metastatic involvement; however, an MRI could not be performed due to the patient's clinical situation and subsequent exitus. The water restriction test confirmed the diagnostic suspicion of central diabetes insipidus. Medical treatment with desmopressin was started to avoid fluid depletion with improvement of natremia figures. It represents an exceptional case of central diabetes insipidus masked by severe hyponatremia in a patient with metastatic lung adenocarcinoma without initial evidence of pituitary metastatic involvement by $\mathrm{CT}$ imaging in treatment with nivolumab (anti-PD-1 agent). Secondary adrenal insufficiency due to pituitary metastatic involvement and endocrinologic toxicity immunorelated to the new checkpoint inhibitors should be considered as possible etiologic agents of central diabetes insipidus, even with hyponatremia.
\end{abstract}

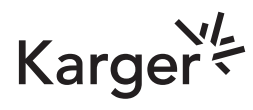




\section{Introduction}

Hyponatremia is an electrolyte disorder frequently encountered in the clinic [1]. Patients with lung cancer may develop hyponatremia through two main endocrinological pathways: syndrome of inappropriate antidiuretic hormone secretion (SIADH) [2] and adrenal insufficiency which is primary or secondary to metastatic involvement [3]. It is known that among the different types of tumors, lung cancer can cause pituitary metastases, although it is uncommon (about 1\%), with the posterior lobe being the most frequently affected due to the irrigation by the pituitary artery [4]. Adrenal insufficiency of tumor etiology should be considered as an alternative diagnosis in patients with lung cancer and hyponatremia.

Regarding the etiopathogenesis of hypernatremia, central diabetes insipidus (DIC), characterized by the partial or total absence of antidiuretic hormone (ADH) and causing a clinical picture consisting of polyuria and polydipsia, should be highlighted as an entity of its own. Most cases are idiopathic, followed by primary or secondary tumors affecting hypophysiothalamic structures $[5,6]$.

On the other hand, immunorelated endocrinopathies such as hypophysitis have been described with the use of checkpoint inhibitors as possible etiopathogenic agents, with an overall incidence of $17 \%$ [7]. We present the exceptional case of a patient with metastatic lung adenocarcinoma under treatment with nivolumab (anti-PD-1 agent) who presented with central diabetes insipidus (DIC) masked by severe hyponatremia. This diagnostic suspicion should not be overlooked when hyponatremia is found in patients with advanced neoplasms.

\section{Case Report}

This case reports a 46-year-old male with no known drug allergies, noninsulin-dependent diabetic and operated for gastric ulcus, and on regular chronic treatment with metformin and methylprednisolone in a descending regimen. He was diagnosed in May 2017 with stage IV adenocarcinoma of the lung due to metastatic involvement at bilateral lung and brain level; 7 lesions (Fig. 1), EGFR mutated (Ex19 deletion). He received holocranial radiotherapy, and in June 2017, it was decided to start treatment with erlotinib with partial response at pulmonary and cerebral level in re-evaluation computed tomography (CT) in October 2017. In February 2018, progression was evidenced, and liquid biopsy was performed objectifying Ex19De mutation and T790M mutation. PD-L1 was 50\%. In March 2018, treatment with osimertinib was started which continued until June 2019 when he presented with progression at pulmonary level. He subsequently underwent several lines of chemotherapy treatment: carboplatin-pemetrexed, gemcitabine monotherapy, paclitaxel-carboplatin-bevacizumab, and vinorelbine. In April 2020, due to new progression at pulmonary and bone level, he started treatment with nivolumab, of which he received 2 cycles. This patient came to the emergency department with symptoms of temporospatial disorientation and dizziness without turning objects lasting 3-4 days, without associated loss of consciousness. He reported an increase in his usual dyspnea without cough or expectoration suggesting signs of infection. Anamnesis by apparatus revealed a daily intake of $9 \mathrm{~L}$ of water with associated sensation of thirst and intense polyuria. No other accompanying symptoms were identified. The patient was conscious, although with slight disorientation in time and space, normoperfused, and tachypneic (28 breaths/min). Rhythmic cardiac auscultation without murmurs and pulmonary auscultation with scattered wheezing were evidenced. The abdomen was soft, depressible, and painless with no evidence of signs of peritoneal irritation. No edema in lower limbs or signs of thrombosis were found. Neurological examination showed slow speech with

\section{Karger'}




\section{Case Reports in Oncology}

\begin{tabular}{l|l}
\hline Case Rep Oncol 2022;15:91-98 \\
\hline DOI: 10.1159/000521608 & $\begin{array}{l}\text { @ 2022 The Author(s). Published by S. Karger AG, Basel } \\
\text { www.karger.com/cro }\end{array}$ \\
\hline
\end{tabular}

Terán Brage et al.: Severe Hyponatremia Masking Central Diabetes Insipidus

Fig. 1. Head MRI: coronal cut; T2 flair; metastatic lesion in the right cerebellar hemisphere.
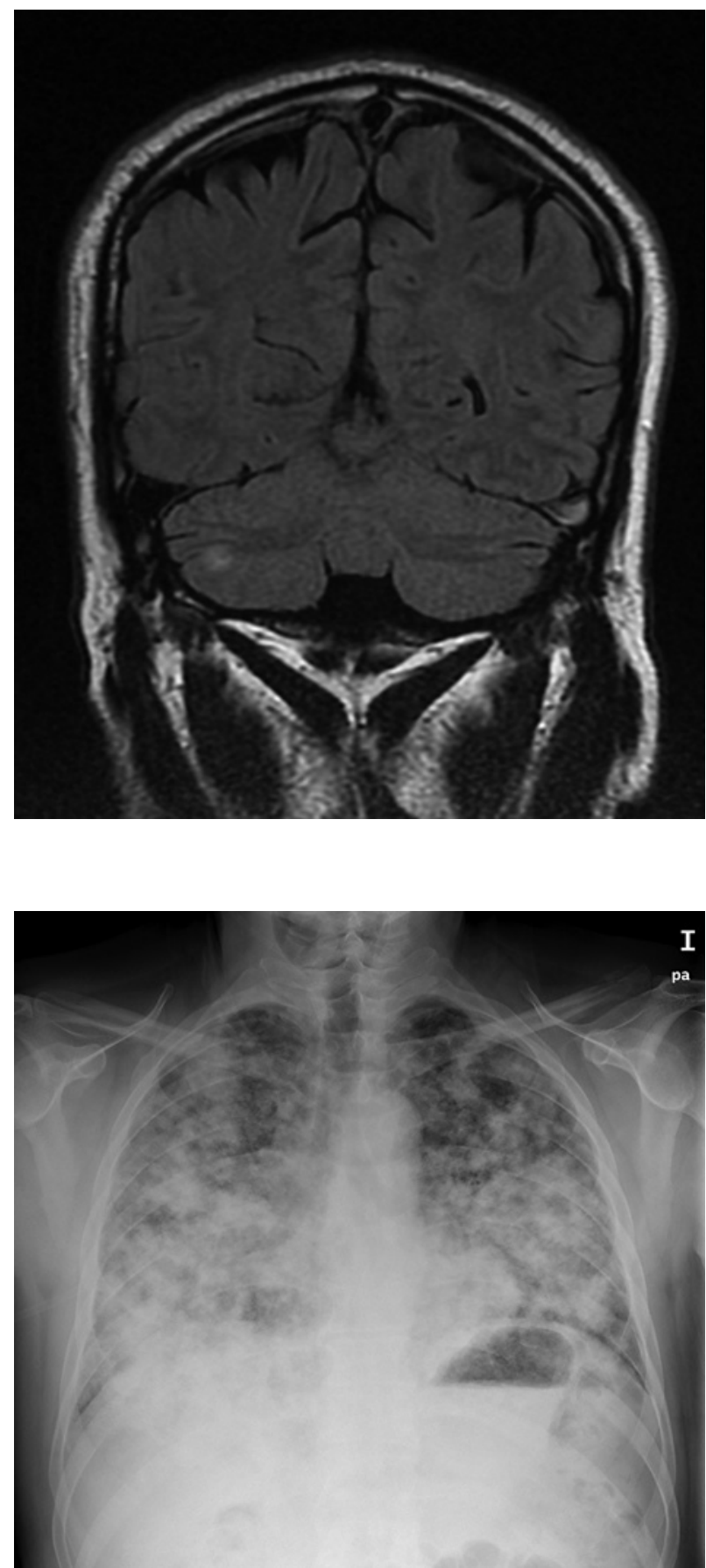

Fig. 2. Chest X-ray on admission: bilateral pulmonary metastases.

\section{I}

\section{.}


Table 1. Results of laboratory examinations performed at admission

\begin{tabular}{lll}
\hline Parameters & Value & Reference range \\
\hline Chemistry & & \\
Hemoglobin, g/dL & 14.5 & $13-18$ \\
Leukocytes & $10.91 \times 10^{3}$ & $4.5-10.8 \times 10^{3}$ \\
Neutrophils & $8.04 \times 10^{3}$ & $1.4-6.5 \times 10^{3}$ \\
Platelets & $283 \times 10^{3}$ & $150-450$ \\
Blood urea nitrogen, mg/dL & 24 & $17-49$ \\
Creatinine, mg/dL & 0.91 & $0.7-1.2$ \\
Uric acid, mg/dL & 4.6 & $3.4-7$ \\
Sodium, mmol/L & 111 & $135-145$ \\
Potassium, mmol/L & 4.8 & $3.5-5.1$ \\
C-reactive protein, mg/dL & 3.33 & $0-0.5$ \\
Plasma osmotic pressure, $\mathrm{mOsm} / \mathrm{kg}$ & 219 & $257-278$ \\
Urine osmolality, mOsm/kg & 89 & $200-800$ \\
Urine sodium, mmol/L & 6 & \\
Encrinology & & \\
Follicle-stimulating hormone (IU/mL), mU/mL & 5.4 & $1.5-12.4$ \\
Luteinizing hormone (IU/mL), mU/mL & 8.6 & $1.7-8.6$ \\
Prolactin, ng/mL & 30.7 & $4.04-15.2$ \\
Testosterone, ng/mL & 89.34 & $249-836$ \\
Beta-estradiol, pg/mL & 16.82 & $7.63-42.6$ \\
Adrenocorticotropic hormone & $<10$ & \\
Insulin-like growth factor-1, ng/mL & 45 & $51.1-221$ \\
Arginine vasopressin, pg/mL & 2.7 & $0-7.6$ \\
Thyroid & & \\
TSH, $\mu$ /mL & 0.553 & $0.93-1.71$ \\
Free T4, ng/mL & 2.29 \\
Free T3, ng/mL & & $2.04-4.4$ \\
\hline
\end{tabular}

Urgent Cranial CT

No evidence of intra or extra-axial bleeding was found. No morphostructural alterations of intracranial expansive lesion or signs of acute ischemia were found and was centered midline.

The analytical control at admission showed sodium $111 \mathrm{mmol} / \mathrm{L}$ and plasma osmolarity $219 \mathrm{mOsm} / \mathrm{kg}$; urine: urine sodium $6 \mathrm{mmol} / \mathrm{L}$ and urinary osmolarity $89 \mathrm{mOsm} / \mathrm{kg}$; thyroid hormones: free T4 $1.75 \mathrm{ng} / \mathrm{dL}(0.93-1.71)$ and TSH $0.553 \mu / \mathrm{mL}$ (0.27-4.2). Complementary examinations of endocrine function revealed values in range of $\mathrm{FSH}, \mathrm{LH}$, and $\mathrm{B}$ estradiol, prolactin $30.7 \mathrm{ng} / \mathrm{dmL}(4.04-15.2)$, and ACTH $<10$. Rest of the parameters are shown in Table 1.

Supportive treatment was started: hypertonic saline 3\% for correction of natremia and oxygen therapy through nasal goggles. According to the patient's symptoms, an urgent cranial CT scan was performed without evidence of intracranial pathology. Given the persistence of severe hypo-osmolar hyponatremia despite the prescribed treatment, assessment and read-

\section{Karger'}


Table 2. Evolution of plasma and urinary sodium levels prior to the start and during the performance of the water restriction test (Miller test)

\begin{tabular}{llllll}
\hline & $\begin{array}{l}\text { June 22, } \\
\end{array}$ & $\begin{array}{l}\text { June 24, } \\
\text { 2020 }\end{array}$ & $\begin{array}{l}\text { June 26, 2020 } \\
\text { (08:25) }\end{array}$ & $\begin{array}{l}\text { June 26, 2020 } \\
(11: 17)\end{array}$ & $\begin{array}{l}\text { June 26, 2020 } \\
(16: 36)\end{array}$ \\
\hline Serum sodium, mmol/L & 111 & 113 & 129 & 131 & 135 \\
Plasma osmotic pressure, mOsm/kg & 219 & 222 & 256 & 258 & 265 \\
Urine osmolality, mmol/L & 6 & 10 & 23 & 29 & 41 \\
Osmolaridad urinaria, mOsm/kg & 89 & 107 & 273 & 315 & 515 \\
\hline
\end{tabular}

justment of treatment was requested by the Nephrology Department. The patient was screened for central diabetes insipidus by the Miller test, showing a weight loss of $8 \mathrm{~kg}$ in a period of $5 \mathrm{~h}$ : from $85.5 \mathrm{~kg}(06: 30 \mathrm{~h})$ to $77.5 \mathrm{~kg}(11: 30 \mathrm{~h})$. After administration of vasopressin (11:00; June 26,2020$)$, an increase $(>50 \%)$ in urinary concentration was observed (Table 2 ), confirming the diagnostic suspicion, and treatment with desmopressin (4 mg iv) was started with improvement of the clinical symptoms, decrease in polyuria, increase in urinary osmolarity, and normalization of natremia levels (Table 2: evolution of plasma and urinary sodium levels). Cranial MRI was requested to rule out morphostructural alteration causing the condition, but due to worsening respiratory symptoms with increased oxygen requirements, the MRI was suspended. Despite supportive treatment, corticotherapy, and intensification of depletive treatment, the patient evolved unfavorably, being exitus.

\section{Clinical Judgment}

This case shows central diabetes insipidus (DIC) masked by severe hyponatremia in a patient with a history of metastatic lung adenocarcinoma.

\section{Discussion}

Central diabetes insipidus can be a secondary manifestation of cancer and is often accompanied by elevated sodium levels [5]. We present an unusual case of hyponatremia as an initial manifestation of central diabetes insipidus as a paraneoplastic manifestation of lung adenocarcinoma. In a review of the literature published to date, Watanabe et al. [8] describe for the first time a case of central diabetes insipidus masked by severe hyponatremia in a patient with pituitary metastases from a lung adenocarcinoma. Ectopic production of ADH by tumor cells plays a causal role in the development of hyponatremia in these patients.

Alterations in natremia are frequent alterations in patients with lung cancer. Hyponatremia correlates strongly with elevated plasma ADH levels as a paraneoplastic manifestation, with SIADH being the initial suspicion in any patient with lung cancer and hyponatremia $[2,9]$.

In the case presented here, the initial diagnostic hypothesis was also SIADH; however, with the data provided in the anamnesis (polyuria and high fluid intake), the deficient increase in urinary osmolarity after water restriction, as well as the possible metastatic pituitary involvement, made other etiological diagnoses such as diabetes insipidus feasible.

Through the subsequent water restriction test (Miller's test) that was performed, a remarkable weight loss with persistent polyuria was evidenced. After administration of vasopressin, an increase ( $>50 \%$ ) in urinary concentration was observed (Table 2), confirming the diagnosis of central diabetes insipidus. 
We know that central or neurogenic diabetes insipidus (CID) is characterized by the absence of $\mathrm{ADH}$, resulting in a variable degree of polyuria and hypernatremia [10]. The absence of ADH may be caused by a disorder acting at one or more sites involved in ADH secretion: the hypothalamic osmoreceptors, the supraoptic nucleus, paraventricular, or in the superior portion of the supraoptic tract.

Patients with untreated central diabetes insipidus typically present with polyuria, nocturia, and, due to the initial elevation of serum sodium and osmolarity, polydipsia, as in the case presented here $(9 \mathrm{~L} /$ day). The presence of neurological symptoms related to the underlying neurological disease is also frequent.

The serum sodium concentration in patients with DIC is normally in the high range of normality, necessary as an initial thirst stimulus to replace water loss. Hence, the exceptionality of the case, severely decreased serum sodium levels ( $\mathrm{Na} 111 \mathrm{mmol} / \mathrm{L}$ ) in the acute situation due to compensatory water intake. In analytical controls prior to the acute event, this patient had sodium values of $146 \mathrm{mmol} / \mathrm{L}$. Different degrees of hypernatremia can be observed in patients with inability to express thirst sensation, mostly in cases of central nervous system lesions.

The most frequent causes of DIC are idiopathic, primary or secondary to tumors, infiltrative diseases, surgical interventions, or trauma. Approximately 30-50\% of cases of DIC are idiopathic, being associated with the destruction of hormone-secreting cells in the hypothalamic nucleus, by an autoimmune mechanism with significant inflammatory lymphocytic reaction in the pituitary stalk and posterior pituitary [11]. The association of primary or secondary tumors due to metastatic involvement at brain level (mainly lung cancer, lymphomas, or leukemias) involving structures at the level of the hypothalamic-pituitary-adrenal axis has been described [12].

Considering the case presented and taking into account the history of metastatic lung adenocarcinoma, as a first diagnostic suspicion, a cranial CT scan was performed demonstrating the absence of morphostructural alterations at that level (hypothalamic-pituitaryadrenal axis). However, although the MRI study is useful in the identification of hyperintensities in the posterior pituitary or pituitary stalk, this test could not be performed due to clinical deterioration and subsequent exitus of the patient, and it was not possible to rule out secondary brain involvement causing the clinical picture.

In the differential diagnosis and genesis of hyponatremia, it is of interest to comment on adrenal insufficiency secondary to infiltrative processes at the level of the pituitary-adrenal axis and exhaustive complementary examinations of endocrinological function [13]. Considering the patient presented, the hormonal study showed no evidence of panhypopituitarism; FSH and LH were in normal range except for prolactin levels of $30.7 \mathrm{ng} / \mathrm{dmL}$ (4.04-15.2) and ACTH $<10$ (justified by chronic corticosteroid intake).

The patient presented was being treated with nivolumab; a checkpoint inhibitor (antiPD-1) currently widely used on various types of tumors. The introduction of immunotherapy in the treatment of advanced lung cancer is offering very encouraging results in terms of overall survival. However, endocrinopathies immunorelated to the use of these agents have been reported in the literature $[7,14]$.

Immunotherapy-associated hypophysitis differs in several aspects from primary hypophysitis and depends on the type of the agent used. Their pathogenesis remains unknown, although CTLA- 4 and PD-1 expression in pituitary cells may play a role. About $10 \%$ of cases have been reported in patients treated with anti-CTLA4 agents (ipilimumab) 2-3 months after initiation of the immunotherapy agent. Cases have been reported with the combination of ipilimumab (anti-CTLA4) and nivolumab (anti-PD-1) and in rare cases associated with anti-PD-1 and antiPD-L1 monotherapy, characterized by a later onset and less symptomatic presentation. Given the lack of evidence of intracranial structural pathology, the possibility of endocrinologic toxicity to treatment with anti-PD-1 agent (nivolumab) in our patient arises, although the onset of the clinical picture at the beginning of treatment (2nd cycle) makes this less likely to be the cause.

\section{Karger'}




\section{Conclusion}

Central diabetes insipidus represents a paraneoplastic manifestation in patients with lung cancer and can occur with any type of natremia, so it requires a high degree of initial suspicion. This study alerts to the presence of central diabetes insipidus masked even with hypo-osmotic hyponatremia data. In this case, a probable intracranial metastatic involvement not evidenced by CT imaging or immunotoxicity associated with nivolumab treatment could have contributed to its genesis.

\section{Statement of Ethics}

Written informed consent has been obtained from the patient's family for publication of the details of their medical case. The study is exempt from ethics committee approval since it is a case report at the University Hospital of Salamanca.

\section{Conflict of Interest Statement}

The authors have no conflicts of interest to declare.

\section{Funding Sources}

No funding was received for this study.

\section{Author Contributions}

T.B.E., H.B.M., and N.J.MB were responsible for literature review, data collection, and manuscript writing. H.B.M. collaborated in the performance of complementary tests that supported the definitive diagnosis. dB.M.E., V.T.R., and F.S.E. cared for the patient during the chemotherapy treatment. All authors contributed to manuscript review before submission and approved the final version.

\section{Data Availability Statement}

The datasets used and analyzed during the current study are available from the corresponding author on reasonable request. Legal entity responsible for the study: the authors.

\section{References}

1 Hoorn EJ, Zietse R. Diagnosis and treatment of hyponatremia: compilation of the guidelines. J Am Soc Nephrol. 2017 May;28(5):1340-9.

2 Gulsin GS, Jacobs ML, Gohil S, Thomas A, Levy M. Competing interests in a lung cancer with metastasis to the pituitary gland: syndrome of inappropriate ADH secretion versus diabetes insipidus. Oxf Med Case Reports. 2016 Jun 1;2016(6):125-9.

3 Chabre 0, Goichot B, Zenaty D, Bertherat J. Group 1. Epidemiology of primary and secondary adrenal insufficiency: prevalence and incidence, acute adrenal insufficiency, long-term morbidity and mortality. Ann Endocrinol. 2017 Dec;78(6):490-4. 
4 Komninos J, Vlassopoulou V, Protopapa D, Korfias S, Kontogeorgos G, Sakas DE, et al. Tumors metastatic to the pituitary gland: case report and literature review. J Clin Endocrinol Metab. 2004 Feb;89(2):574-80.

5 Rose DB, Post TW. Clinical physiology of acid-base and electrolyte disorders. 5th ed. New York: McGraw-Hill; 2001. p. P751.

6 Matsuda R, Chiba E, Kawana I, Kihara M, Tomiyama M, Ebira H, et al. Central diabetes insipidus caused by pituitary metastasis of lung cancer. Intern Med. 1995 Sep;34(9):913-8.

7 Du Payrat JA, Cugnet-Anceau C, Maillet D, Levy M, Raverot G, Disse E, et al. Hypophysites induites par les immunotherapies anti-néoplasiques. [Checkpoint inhibitor-induced hypophysitis]. Bull Cancer. 2020 Apr;107(4): 490-8.

8 Watanabe M, Yasuda J, Ashida K, Matsuo Y, Nagayama A, Goto Y, et al. Diabetes insipidus hidden by severe hyonatremia: a case of pituitary metastasis of lung adenocarcinoma. Am J Case Rep. 2020 Dec 18;21:e928113.

9 Fiordoliva I, Meletani T, Baleani MG, Rinaldi S, Savini A, Di Pietro Paolo M, et al. Managing hyponatremia in lung cancer: latest evidence and clinical implications. Ther Adv Med Oncol. 2017;9(11):711-9.

10 Sirinvaravong S, Vibhatavata P, Chunharojrith P, Cheunsuchon P, Sriussadaporn S. Diabetes insipidus and panhypopituitarism as a first presentation of silent adenocarcinoma of lung: a case report and literature review. BMC Endocr Disord. 2019 Oct 29;19(1):114.

11 Pivonello R, De Bellis A, Faggiano A, Di Salle F, Petretta M, Di Somma C, et al. Central diabetes insipidus and autoimmunity: relationship between the occurrence of antibodies to arginine vasopressin-secreting cells and clinical, immunological, and radiological features in a large cohort of patients with central diabetes insipidus of known and unknown etiology. J Clin Endocrinol Metab. 2003 Apr;88(4):1629-36.

12 Castle-Kirszbaum M, Goldschlager T, Ho B, Wang YY, King J. Twelve cases of pituitary metastasis: a case series and review of the literature. Pituitary. 2018 Oct;21(5):463-73.

13 Martin-Grace J, Dineen R, Sherlock M, Thompson CJ. Adrenal insufficiency: physiology, clinical presentation and diagnostic challenges. Clin Chim Acta. 2020 Jun; 505:78-91.

14 Torino F, Corsello SM, Salvatori R. Endocrinological side-effects of immune checkpoint inhibitors. Curr Opin Oncol. 2016 Jul;28(4):278-87. 